\title{
Efficacy, Tolerance, and Plasma Levels of Abiraterone and Its Main Metabolites in a Patient With Metastatic Castration-resistant Prostate Cancer With a Hepatic Transplant
}

\author{
Merel van Nuland, ${ }^{1,2}$ Julie M. Janssen, ${ }^{1,2}$ Bart van Hoek, ${ }^{4}$ Hilde Rosing, ${ }^{1}$ \\ Jos H. Beijnen, ${ }^{1,2,3}$ André M. Bergman ${ }^{5}$
}

\section{Clinical Practice Points}

- Abiraterone acetate, which is metabolized in the liver, is a well-established treatment option for patients with metastatic castration-resistant prostate cancer. The impact of hepatic impairment on exposure to abiraterone was well-studied during registration studies, and abiraterone acetate is contraindicated for patients with severe hepatic impairment. Patients with a liver transplant are prone to impaired liver functions and use medication that may affect drug metabolism. However, no efficacy, tolerance, and pharmacokinetic data have been published on abiraterone treatment in patients who have undergone liver transplants.

- In this case report, we established plasma concentrations of abiraterone and its major metabolites, $\Delta(4)$ abiraterone, abiraterone $\mathrm{N}$-oxide sulfate, and abiraterone sulfate, in a patient with metastatic castration-resistant prostate cancer with a hepatic transplant who was treated with abiraterone in a reduced dose of $500 \mathrm{mg}$ daily.

- Treatment was effective and well-tolerated, and plasma concentrations were above the suggested trough concentration threshold of $8.4 \mathrm{ng} / \mathrm{mL}$. Moreover, the exposure to immunosuppressive drugs was within expected therapeutic ranges.

- From this case, we conclude that abiraterone actetate seems to be a feasible and safe treatment strategy for patients with a hepatic transplant. However, further clinical studies should be performed in order to confirm these findings.

Clinical Genitourinary Cancer, Vol. 17, No. 5, e893-6 (c) 2019 Elsevier Inc. All rights reserved. Keywords: Hepatotoxicity, Liver transplant, Metabolite, Prostate cancer, Therapeutic drug monitoring

\section{Introduction}

Abiraterone acetate (Zytiga) is a $17 \alpha$-hydroxylase/C17,20-lyase (CYP17) inhibitor, which prevents the production of tumorstimulating androgens such as testosterone. Abiraterone acetate was

\footnotetext{
${ }^{1}$ Department of Pharmacy and Pharmacology

${ }^{2}$ Division of Pharmacology, The Netherlands Cancer Institute, Amsterdam, The Netherlands

${ }^{3}$ Division of Pharmacoepidemiology and Clinical Pharmacology, Utrecht Institute for Pharmaceutical Sciences, Utrecht University, Utrecht, The Netherlands

${ }^{4}$ Department of Gastroenterology and Hepatology, Leiden University Medical Center, Leiden, The Netherlands

${ }^{5}$ Division of Medical Oncology, The Netherlands Cancer Institute, Amsterdam, The Netherlands
}

Submitted: May 8, 2019; Accepted: May 26, 2019; Epub: May 30, 2019

Address for correspondence: Merel van Nuland, PharmD, The Netherlands Cancer Institute, Plesmanlaan 121, 1066 CX Amsterdam

E-mail contact: m.v.nuland@nki.nl registered for treatment of metastatic castration-resistant prostate cancer (mCRPC) as it improves overall survival and progression-free survival in this patient population compared with placebo. ${ }^{1,2}$

After oral ingestion, abiraterone acetate is rapidly deacetylated into the active substance abiraterone. ${ }^{3}$ Further hepatic metabolism of abiraterone is extensive, and the inactive metabolites abiraterone $\mathrm{N}$ oxide sulfate and abiraterone sulfate are formed. ${ }^{3,4}$ More recently, the active metabolite $\Delta(4)$-abiraterone (D4A) was discovered, which is formed by conversion of abiraterone by the enzyme $3 \beta$-hydroxysteroiddehydrogenase., ${ }^{5,6}$ D4A not only blocks CYP17, but also inhibits multiple steroidic enzymes and blocks the androgen receptor, ${ }^{6,7}$ which makes it likely that D4A is even more potent than abiraterone.

Clinical studies have shown that approximately $2 \%$ of patients using abiraterone acetate show liver function test elevations. Therefore, dose modifications are recommended for patients who develop hepatotoxicity while on treatment. ${ }^{3}$ In a dedicated hepatic impairment study, 


\section{Abiraterone Acetate in a Patient With a Hepatic Transplant}

exposure to abiraterone (1000 mg once daily [QD]) was similar in subjects with mild hepatic impairment (Child-Pugh classification A; $\mathrm{n}=8$ ) and in subjects with normal hepatic function. However, subjects with moderate hepatic impairment (Child-Pugh classification $B ; n=8$ ) and severe hepatic impairment (Child-Pugh classification $\mathrm{C} ; \mathrm{n}=8$ ) had higher exposure, a higher maximum concentration $\left(\mathrm{C}_{\max }\right)$, and a longer elimination half-life $\left(\mathrm{t}_{1 / 2}\right)$ compared with subjects with a normal hepatic function. From this study, it was concluded that a dose reduction to $250 \mathrm{mg}$ is recommended in patients with moderate hepatic impairment, whereas abiraterone acetate is contraindicated in patients with severe hepatic impairment. ${ }^{8}$

Although the impact of hepatic impairment on abiraterone exposure was studied during registration, no treatment adjustments are provided for patients with cancer with a hepatic transplant. Patients with a functional liver transplant are at risk to develop impaired liver functions and use medication that may affect drug pharmacokinetics. ' We here present a case of a patient with mCRPC with a hepatic transplant who was treated with abiraterone acetate. Plasma concentrations of abiraterone and its major metabolites were assessed, and concomitant treatment with immunosuppressive agents cyclosporin and mycophenolic acid was evaluated.

\section{Case Report}

A 76-year-old male with a history of localized prostate cancer and a hepatic transplant presented with mCRPC. The liver transplant was placed in 2006 because of liver failure owing to primary biliary cirrhosis with Child-Pugh classification B/C. After a first rejection of the liver allograft, a second liver transplant was placed in the same year. To date, the liver function of this second transplant is adequate.

The patient was first diagnosed with $\mathrm{cT} 1 \mathrm{cNxM} 0$ prostate cancer and a Gleason score of 6 in 2000 and was initially treated with external beam radiotherapy to the prostate. In 2017, bone metastases were found, and androgen deprivation treatment (goserelin $10.8 \mathrm{mg}$ subcutaneously every 3 months) was initiated. mCRPC was established in 2018 when serum prostate-specific antigen (PSA) levels progressed under suppressed serum testosterone levels $(<1.7$ $\mathrm{nmol} / \mathrm{L}$ ). Patient characteristics are given in Table 1 . Concomitant immunosuppressive medication consisted of cyclosporin $(50 \mathrm{mg}$ twice daily [BID]), mycophenolic acid (1500 mg BID), and other medication, including denosumab (70 mg every month), calcium carbonate/cholicalciferol (1.25g/800 IE QD), ursodeoxycholic acid (250 mg BID), and pantoprazole (20 mg BID). In October 2018, progressive bone metastases were established, and abiraterone acetate in combination with prednisone $(5 \mathrm{mg}$ BID) under fasting conditions was initiated. Because the tolerance of abiraterone in a patient with a liver transplant could not be predicted, abiraterone treatment was initiated at a reduced dose of $500 \mathrm{mg}$ QD. Abiraterone acetate treatment was well-tolerated with an initial drop in serum PSA from 119 to $36.6 \mathrm{ng} / \mathrm{mL}$. However, 2 months after the start of therapy, the PSA level slightly increased, and prednisone was replaced by dexamethasone $(0.5 \mathrm{mg} \mathrm{QD})$ to re-induce abiraterone sensitivity. ${ }^{10}$ During abiraterone and dexamethasone treatment, the PSA further declined to $28.8 \mathrm{ng} / \mathrm{mL}$. Furthermore, a decrease in serum alkaline phosphatase levels was observed, which may serve as a biomarker for the extent of bone metastasis. ${ }^{11}$

During treatment, plasma concentrations of abiraterone and its main metabolites D4A, abiraterone $\mathrm{N}$-oxide sulfate, and abiraterone sulfate were measured using validated liquid chromatographytandem mass spectrometry methods. ${ }^{12,13}$ Plasma trough concentrations $\left(\mathrm{C}_{\text {trough }}\right)$ of abiraterone and its metabolites were calculated using Equation 1, in order to compare the individual plasma levels with $\mathrm{C}_{\text {trough }}$ concentrations from literature. Plasma concentrations were above the suggested target concentration of $8.4 \mu \mathrm{g} / \mathrm{L},{ }^{14}$ and thus abiraterone remained at $50 \%$ of the dose throughout treatment. Table 1 shows the exposure to cyclosporin and mycophenolic acid as well as plasma concentrations of abiraterone and its metabolites during treatment. Figure 1 visualizes the active drug and metabolite concentrations along with PSA levels. ${ }^{15}$

$$
C_{\text {trough }}=C_{T A D} * 0.5^{\frac{24-T A D}{t_{1}}}
$$

Equation 1

Wherein $\mathrm{C}_{\text {trough }}$ is the calculated trough concentration in $\mu \mathrm{g} / \mathrm{L}$ and $\mathrm{C}_{\mathrm{TAD}}$ the measured concentration in $\mu \mathrm{g} / \mathrm{L}$ at the recorded time after abiraterone acetate dosing (TAD), given in hours. Trough concentrations were calculated using the following $\mathrm{t}_{1 / 2}: 15$ hours for abiraterone, abiraterone sulphate, and D4A, and 21.6 hours for abiraterone $\mathrm{N}$-oxide sulphate, respectively. ${ }^{4}$ Furthermore, exposure to cyclosporin and mycophenolic acid was monitored using a limited sampling strategy: drug concentrations were determined at $0,1,2$, and 3 hours after administration, and the area under the curve was estimated from 0 to 12 hours $\left(\mathrm{AUC}_{0-12}\right) .{ }^{16}$

\section{Discussion}

Abiraterone acetate treatment is an effective treatment option for patients with mCRPC. Although it is known that the exposure to abiraterone is higher in patients with moderate and severe hepatic impairment compared with subjects with a normal hepatic function, ${ }^{8}$ no data has been published on the exposure to abiraterone in hepatic transplant recipients. In this case report, we describe the treatment with abiraterone of a patient with mCRPC who had undergone a previous liver transplant.

Treatment options for mCRPC, apart from abiraterone acetate, include enzalutamide and docetaxel. Enzalutamide is a strong cytochrome P450 3A4 (CYP3A4) inducer and was therefore not recommended in combination with cyclosporin, whereas docetaxel may elevate hepatic markers and is contraindicated for patients with hepatic dysfunction. ${ }^{17,18}$ Therefore, this patient was treated with abiraterone acetate, which was tolerated well at the administered dose of $500 \mathrm{mg}$ QD, and, most importantly, no hepatotoxicity was observed. Plasma concentrations were determined at steady-state, with the mean trough concentration of abiraterone being $33 \mu \mathrm{g} /$ L. In a prospective observational trial in patients with $\mathrm{mCRPC}$, a relationship was found between abiraterone trough levels and PSA response. PSA-responders $(\mathrm{n}=38)$ had significantly higher plasma concentrations of abiraterone compared with non-responders $(\mathrm{n}=$ 23) (12.0 vs. $8.0 \mu \mathrm{g} / \mathrm{L} ; P=.0015)$. By receiver operating characteristics analysis, a minimum $\mathrm{C}_{\text {trough }}$ of $8.4 \mu \mathrm{g} / \mathrm{L}$ was defined as a target for exposure to abiraterone. ${ }^{14}$ In our case, the calculated trough concentrations were above this threshold, indicating adequate exposure to abiraterone with $50 \%$ of the dose.

The mean trough concentration of the active metabolite D4A $(1.5 \mu \mathrm{g} / \mathrm{L})$ was in line with the mean $\mathrm{C}_{\text {trough }}$ in a previously reported study $(1.6 \mu \mathrm{g} / \mathrm{L} ; \mathrm{n}=36) .{ }^{19}$ Mean trough concentrations of 
Table 1 Patient Characteristics at Baseline and During Treatment

\begin{tabular}{|c|c|c|c|c|c|}
\hline Parameter & Baseline & 1 Month & 2 Months & 3 Months & 4 Months \\
\hline Dose, mg (QD) & - & 500 & 500 & 500 & 500 \\
\hline \multicolumn{6}{|l|}{ Plasma concentrations, $\mu \mathrm{g} / \mathrm{L}^{\mathrm{a}}$} \\
\hline Abiraterone & - & $47.8(131)$ & $30.3(70.1)$ & $20.9(45.8)$ & - \\
\hline D4A & - & $2.00(5.42)$ & $1.40(3.31)$ & $1.00(2.15)$ & - \\
\hline Abiraterone $\mathrm{N}$-oxide sulfate & - & $3619(7440)$ & $5609(10,200)$ & $5991(10,500)$ & - \\
\hline Abiraterone sulfate & - & $5907(16,200)$ & 3966 (9160) & $3123(6850)$ & - \\
\hline \multicolumn{6}{|l|}{$\mathrm{AUC}_{0-12}, \mathrm{mg} / \mathrm{L} \bullet \mathrm{h}$} \\
\hline Cyclosporin & 1.42 & - & - & 1.08 & - \\
\hline Mycophenolic acid & 48 & - & - & 34 & \\
\hline $\mathrm{PSA}, \mu \mathrm{g} / \mathrm{L}$ & 119.2 & 36.61 & 39.65 & 46.07 & 28.76 \\
\hline \multicolumn{6}{|l|}{ Kidney function } \\
\hline Creatinine, $\mu \mathrm{mol} / \mathrm{L}$ & 100 & 100 & 87 & 99 & 90 \\
\hline eGFR (MDRD-4) & 63 & 63 & 74 & 64 & 71 \\
\hline \multicolumn{6}{|l|}{ Hepatic markers } \\
\hline Bilirubin, total, $\mu \mathrm{mol} / \mathrm{L}$ & 18 & 11 & 13 & 14 & 15 \\
\hline Bilirubin, direct, $\mu \mathrm{mol} / \mathrm{L}$ & - & - & - & - & 7 \\
\hline ASAT, U/L & 33 & 21 & 24 & 19 & 26 \\
\hline ALAT, U/L & 20 & 21 & 18 & 15 & 13 \\
\hline$\gamma$-GT, U/L & 141 & 89 & 70 & 49 & 56 \\
\hline Alkaline phosphatase, U/L & 1153 & 2326 & 1045 & 419 & 359 \\
\hline Change from baseline, $\quad \%$ & - & 202 & 91 & 36 & 31 \\
\hline Albumin, $g / L$ & 41 & 41 & 45 & 43 & 45 \\
\hline Total protein, g/L & 73 & 69 & 71 & 71 & 69 \\
\hline $\mathrm{LDH}, \mathrm{U} / \mathrm{L}$ & 182 & 148 & 179 & 168 & 176 \\
\hline APT, sec & 28 & - & - & - & 29 \\
\hline PT-INR & 1.1 & - & - & - & 1.1 \\
\hline Haptoglobulin, g/L & - & - & - & - & 1.0 \\
\hline t-Amylase, U/L & - & - & - & - & .61 \\
\hline Cholesterol, mmol/L & - & - & - & - & 4.7 \\
\hline Tryglycerides, mmol/L & - & - & - & - & 1.0 \\
\hline Ammoniak, $\mu \mathrm{mol} / \mathrm{L}$ & - & - & - & - & 32 \\
\hline Testosterone, nmol/L & - & $<0.025$ & $<0.025$ & $<0.025$ & $<0.025$ \\
\hline
\end{tabular}

Abbreviations: ALAT = alanine aminotransferase; APT = activated partial thromboplastin time; ASAT = aspartate aminotransferase; AUC = area under the curve; D4A = $\Delta(4)$-abiraterone; eGFR = estimated glomerular filtration rate; $\gamma$-GT = gamma-glutamyltransferase; LDH = lactate dehydrogenase; MDRD = Modification of Diet in Renal Disease; PSA = prostate-specific antigen; PT-INR = prothrombin time-international normalized ratio.

${ }^{a}$ Calculated trough concentrations and the originally measured plasma concentrations in parentheses.

abiraterone $\mathrm{N}$-oxide sulfate and abiraterone sulfate were $5073 \mu \mathrm{g} / \mathrm{L}$ and $4332 \mu \mathrm{g} / \mathrm{L}$, respectively. To our knowledge, no abiraterone $\mathrm{N}$ oxide sulfate and abiraterone sulfate concentrations have been reported in patients with $\mathrm{mCRPC}$ with which to compare these data.

Abiraterone is a strong inhibitor of CYP2D6 and a mild inhibitor of CYP2C8, while being metabolized by SULT2A1, CYP3A4, and $3 \beta$-hydroxysteroid-dehydrogenase. Furthermore, the major metabolites, abiraterone sulfate and abiterone $\mathrm{N}$-oxide sulfate, inhibit the uptake transporter organic-anion-transporting polypeptide 1B1 (OATP1B1) in vitro. ${ }^{20}$ Cyclosporin inhibits breast cancer resistant protein (BCRP) and OATP1B and is substrate for CYP3A4 and Pglycoprotein (P-gp), whereas mycophenolic acid is predominantly metabolized by uridine 5'-diphospho-glucuronosyltransferase 1A9 (UGT1A9) and is substrate for transporters OATP, BCRP, and multi-drug resistant associated protein 2 (MRP2). ${ }^{21,22}$ Based on this information, OATP inhibition by abiraterone metabolites and cyclosporin may affect mycopholic acid exposure. However, this putative interaction did not affect treatment of our patient, as exposure to mycophenolic acid was well-tolerated, and the AUC was within the therapeutic range $\left(30-60 \mathrm{mg} / \mathrm{L} \bullet \mathrm{h}^{16}\right)$. The combination of cyclosporin and mycophenolic acid was given to preserve renal function in this patient with 1 kidney and some renal insufficiency, as cyclosporin may induce nephrotoxicity. The cyclosporin dose was based on liver enzymes, resulting in an AUC of $1.08 \mathrm{mg} / \mathrm{L} \bullet \mathrm{h}$.

\section{Conclusion}

In this case report, a patient with mCRPC with a hepatic transplant was treated with abiraterone acetate at a reduced dose of $500 \mathrm{mg}$ QD. Plasma levels of abiraterone and its active metabolite D4A were similar to those observed in patients with mCRPC without a hepatic 
Figure 1 Plasma Concentrations of Abiraterone and D4A, Including PSA Levels in a Patient With a Hepatic Transplant Treated With Abiraterone Acetate (500 mg QD). *Prednisone Was Replaced by Dexamethasone, Resulting in a PSA Decline

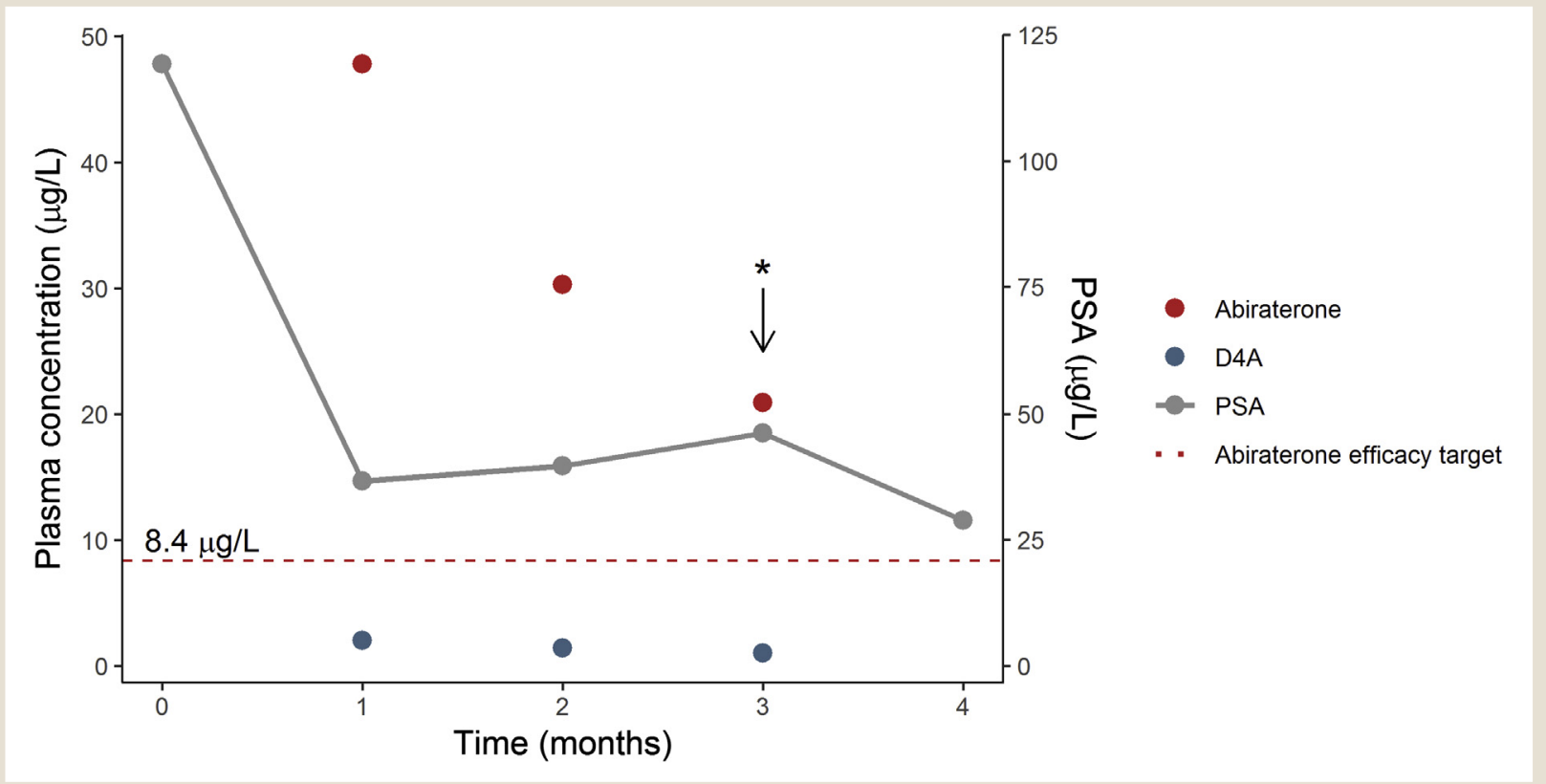

Abbreviations: $\mathrm{D} 4 \mathrm{~A}=\Delta(4)$-abiraterone; PSA $=$ prostate-specific antigen; $\mathrm{QD}=$ once daily.

transplant, no clinical relevant toxicity was observed, and exposure to immunosuppressive drugs mycophenolic acid and cyclosporin were within expected therapeutic ranges. The patient responded well to the treatment, showing a PSA decrease of $>50 \%$. It can thus be concluded that treatment with abiraterone acetate at a $50 \%$ reduced dose seems feasible and safe for hepatic transplant recipients. It is, however, recommended to monitor liver functions and plasma concentrations of abiraterone during treatment. Further clinical studies should be performed in order to confirm these findings.

\section{Disclosure}

The authors have stated that they have no conflicts of interest.

\section{References}

1. de Bono JS, Logothetis CJ, Molina A, et al, COU-AA-301 Investigators. Abiraterone and increased survival in metastatic prostate cancer. $N$ Engl J Med 2011; 364:1995-2005.

2. Ryan CJ, Smith MR, de Bono JS, et al, COU-AA-302 Investigators. Abiraterone in metastatic prostate cancer without previous chemotherapy. N Engl J Med 2013; 368:138-48.

3. United States Food and Drug Administration. Clinical pharmacology and biopharmaceutics review: Zytiga (abiraterone acetate). 2010. p. 1-86. Available at: https:// www.accessdata.fda.gov/drugsatfda_docs/nda/2011/202379orig1s000clinpharmr.pdf. Accessed: January 10, 2019.

4. Acharya M, Gonzalez M, Mannens G, et al. A phase I, open-label, single-dose, mass balance study of 14C-labeled abiraterone acetate in healthy male subjects. Xenobiotica 2013; 43:379-89.

5. Li Z, Alyamani M, Li J, et al. Redirecting abiraterone metabolism to fine-tune prostate cancer anti-androgen therapy. Nature 2016; 533:547-51.

6. Li Z, Bishop AC, Alyamani M, et al. Conversion of abiraterone to D4A drives antitumour activity in prostate cancer. Nature 2015; 523:347-51.

7. Emamekhoo H, Li Z, Sharifi N. Clinical significance of D4A in prostate cancer therapy with abiraterone. Cell Cycle 2015; 14:3213-4.

8. Marbury T, Lawitz E, Stonerock R, et al. Single-dose pharmacokinetic studies of abiraterone acetate in men with hepatic or renal impairment. J Clin Pharmacol 2014; 54:732-41.
9. Parikh ND, Levitsky J. Hepatotoxicity and drug interactions in liver transplant candidates and recipients. Clin Liver Dis 2013; 17:737-47, x-xi.

10. Lorente D, Omlin A, Ferraldeschi R, et al. Tumour responses following a steroid switch from prednisone to dexamethasone in castration-resistant prostate cancer patients progressing on abiraterone. Br J Cancer 2014; 111:2248-53.

11. Armstrong AJ, Febbo PG. Using surrogate biomarkers to predict clinical benefit in men with castration-resistant prostate cancer: an update and review of the literature. Oncologist 2009; 14:816-27.

12. van Nuland M, Hillebrand MJX, Rosing $H$, Schellens JHM, Beijnen JH. Development and validation of an LC-MS/MS method for the simultaneous quantification of abiraterone, enzalutamide, and their major metabolites in human plasma. Ther Drug Monit 2017; 39:243-51.

13. van Nuland M, Rosing H, de Vries J, Ovaa H, Schellens JHM, Beijnen JH. An LC-MS/MS method for quantification of the active abiraterone metabolite $\Delta(4)$ abiraterone (D4A) in human plasma. J Chromatogr B Analyt Technol Biomed Life Sci 2017; 1068-9:119-24.

14. Carton E, Noe G, Huillard O, et al. Relation between plasma trough concentration of abiraterone and prostate-specific antigen response in metastatic castrationresistant prostate cancer patients. Eur J Cancer 2017; 72:54-61.

15. Wang Y, Chia YL, Nedelman J, Schran H, Mahon F-X, Molimard M. A therapeutic drug monitoring algorithm for refining the imatinib trough level obtained at different sampling times. Ther Drug Monit 2009; 31:579-84.

16. van Gelder T, Le Meur Y, Shaw LM, et al. Therapeutic drug monitoring of mycophenolate mofetil in transplantation. Ther Drug Monit 2006; 28:145-54.

17. United States Food and Drug Administration. Clinical pharmacology and biopharmaceutics review: Xtandi (Enzalutamide). Silver Spring (MD). 2012. p. 1-75. Available at: https://www.accessdata.fda.gov/drugsatfda_docs/nda/2012/203415 Orig1s000ClinPharmR.pdf. Accessed: January 10, 2019.

18. Minami H, Kawada K, Sasaki $Y$, et al. Population pharmacokinetics of docetaxel in patients with hepatic dysfunction treated in an oncology practice. Cancer Sci 2009; 100:144-9.

19. Blanchet B, Carton E, Alyamani M, et al. A PK/PD study of Delta-4 abiraterone metabolite in metastatic castration-resistant prostate cancer patients. Pharmacol Res 2018; 136:56-61.

20. European Medicines Agency (EMA). Zytiga-H-C-2321-PSUV-19. European public assessment report: scientific conclusions and grounds recommending the variation to the terms of the marketing authorisation. 2014. p. 1-11. Available at: https://www.ema.europa.eu/en/documents/scientific-conclusion/zytiga-h-c-2321psuv-0017-epar-scientific-conclusions-grounds-recommending-variation-terms-ma rketing_en.pdf. Accessed: April 29, 2019.

21. Staatz CE, Tett SE. Clinical pharmacokinetics and pharmacodynamics of mycophenolate in solid organ transplant recipients. Clin Pharmacokinet 2007; 46:13-58.

22. Fahr A. Cyclosporin clinical pharmacokinetics. Clin Pharmacokinet 1993; 24: $472-95$. 